\title{
HCN Subunit-Specific and cAMP-Modulated Effects of Anesthetics on Neuronal Pacemaker Currents
}

\author{
Xiangdong Chen, ${ }^{1}$ Jay E. Sirois, ${ }^{2}$ Qiubo Lei, ${ }^{1}$ Edmund M. Talley, ${ }^{1}$ Carl Lynch III, ${ }^{2}$ and Douglas A. Bayliss ${ }^{1,2}$ \\ Departments of ${ }^{1}$ Pharmacology and ${ }^{2}$ Anesthesiology, University of Virginia, Charlottesville, Virginia 22908
}

General anesthetics have been a mainstay of surgical practice for more than 150 years, but the mechanisms by which they mediate their important clinical actions remain unclear. Ion channels represent important anesthetic targets, and, although $\mathrm{GABA}_{\mathrm{A}}$ receptors have emerged as major contributors to sedative, immobilizing, and hypnotic effects of intravenous anesthetics, a role for those receptors is less certain in the case of inhalational anesthetics. The neuronal hyperpolarization-activated pacemaker current $\left(I_{h}\right)$ is essential for oscillatory and integrative properties in numerous cell types. Here, we show that clinically relevant concentrations of inhalational anesthetics modulate neuronal $I_{\mathrm{h}}$ and the corresponding HCN channels in a subunit-specific and cAMP-dependent manner. Anesthetic inhibition of $I_{\mathrm{h}}$ involves a hyperpolarizing shift in voltage dependence of activation and a decrease in maximal current amplitude; these effects can be ascribed to $\mathrm{HCN} 1$ and $\mathrm{HCN} 2$ subunits, respectively, and both actions are recapitulated in heteromeric $\mathrm{HCN} 1-\mathrm{HCN} 2$ channels. Mutagenesis and simulations suggest that apparently distinct actions of anesthetics on $V_{1 / 2}$ and amplitude represent different manifestations of a single underlying mechanism (i.e., stabilization of channel closed state), with the predominant action determined by basal inhibition imposed by individual subunit C-terminal domains and relieved by cAMP. These data reveal a molecular basis for multiple actions of anesthetics on neuronal $\mathrm{HCN}$ channels, highlight the importance of proximal $\mathrm{C}$ terminus in modulation of $\mathrm{HCN}$ channel gating by diverse agents, and advance neuronal pacemaker channels as potentially relevant targets for clinical actions of inhaled anesthetics.

Key words: anesthesia; arousal; motoneuron; neuromodulation; pacemaker; thalamus; voltage clamp; cAMP

\section{Introduction}

General anesthetics represent some of the most widely used and useful drugs in clinical medicine; despite their prevalent use, however, the fundamental neural mechanisms by which they exert their actions remain uncertain. It is now generally accepted that specific membrane proteins, particularly ion channels, provide the relevant substrate for anesthetic actions (Franks and Lieb, 1994; Krasowski and Harrison, 1999). The ionotropic $\mathrm{GABA}_{\mathrm{A}}$ receptor has garnered the most attention in this respect, and this emphasis has received elegant validation at the systems level; mice bearing targeted mutations that selectively eliminate anesthetic sensitivity of $\mathrm{GABA}_{\mathrm{A}}$ receptors containing $\beta 2$ or $\beta 3$ subunits display either altered sedative $(\beta 2 \mathrm{~N} 265 \mathrm{~S})$ or anesthetic responses $(\beta 3 \mathrm{~N} 265 \mathrm{M})$ to intravenous anesthetic agents (e.g., etomidate or propofol) (Jurd et al., 2003; Reynolds et al., 2003) (for review, see Rudolph and Mohler, 2004). Interestingly, however, anesthesia induced with inhalational anesthetics (e.g., enflurane) was minimally affected in $\beta 3 \mathrm{~N} 265 \mathrm{M}$ mice, although that muta-

Received Jan. 5, 2005; revised May 15, 2005; accepted May 16, 2005.

This work was supported by United States Public Health Service Grant GM66181 (D.A.B.). We are extremely grateful to Drs. B. Santoro and S. A. Siegelbaum for generous gifts of HCN channel constructs and to Drs. Galen Flynn and William N. Zagotta for insightful comments and suggestions during the course of these studies. We also thank Dr. Eduardo Perozo for help with simulations, Dr. Paula Q. Barrett for input on the manuscript, and Jules Manger and Jackie Washington for assistance with in situ hybridization and gas chromatography.

Correspondence should be addressed to Douglas A. Bayliss, Department of Pharmacology, University of Virginia Health System, P.0. Box 800735, 1300 Jefferson Park Avenue, Charlottesville, VA 22908-0735. E-mail: dab3y@virginia.edu.

DOI:10.1523/JNEUROSCI.1153-05.2005

Copyright $\odot 2005$ Society for Neuroscience $\quad 0270-6474 / 05 / 255803-12 \$ 15.00 / 0$ tion blocks $\mathrm{GABA}_{\mathrm{A}}$ receptor modulation by enflurane (Jurd et al., 2003), suggesting that alternative molecular substrates contribute to actions of inhalation anesthetics (Harrison, 2003; Sonner et al., 2003).

Additional candidate molecular targets for actions of inhalational anesthetics may be found within various classes of subthreshold ion channels that regulate intrinsic neuronal excitability. For example, discovery of the two-pore-domain potassium ion channel family has provided a molecular basis for early observations that "background" $\mathrm{K}^{+}$currents are activated by inhalational anesthetics (Franks and Lieb, 1988; Patel et al., 1999). For TASK (TWIK-related acid-sensitive $\mathrm{K}^{+}$channels) and TREK (TWIK-related $\mathrm{K}^{+}$channel) members of this family, channel domains critical for anesthetic actions have been identified (Patel et al., 1999; Talley and Bayliss, 2002), and, in the case of TREK-1, a knock-out mouse displayed reduced sensitivity to volatile anesthetics (Heurteaux et al., 2004). Likewise, it is now recognized that the HCN family of ion channels gives rise to the cyclic nucleotide-sensitive, hyperpolarization-activated cationic currents, variously called $I_{\mathrm{h}}, I_{\mathrm{f}}$, and $I_{\mathrm{q}}$ (Biel et al., 1999; Santoro and Tibbs, 1999; Kaupp and Seifert, 2001); these currents are renowned for providing a cAMP-modulated, subthreshold pacemaker activity in rhythmically oscillating cells of the heart and brain (DiFrancesco, 1993; Pape, 1996; Clapham, 1998; Lüthi and McCormick, 1998), and, of relevance here, we found that they are strongly modulated by inhalation anesthetics (Sirois et al., 1998, 2002). Although it has been established that HCN channels represent the molecular basis for native $I_{\mathrm{h}}$, it is not certain which 
$\mathrm{HCN}$ subunits mediate anesthetic actions and/or which domains of the proteins are responsible for channel inhibition.

Here, we recorded from neurons in brain slices and from human embryonic kidney 293 (HEK293) cells transfected with HCN1 and HCN2 subunits to characterize anesthetic effects on native neuronal $I_{\mathrm{h}}$ and to determine the molecular basis for inhibition of $I_{\mathrm{h}}$ by inhalation anesthetics. Inhibition of neuronal $I_{\mathrm{h}}$ resulted from a hyperpolarizing shift in the voltage dependence of current activation and a decrease in maximal current amplitude. These two inhibitory effects were observed at clinically relevant anesthetic concentrations, and each was associated with a distinct HCN subunit. The subunit-specific inhibitory actions reflect differential allosteric modulation of the channels by their respective $\mathrm{C}$ termini, and the relative expression of each component of inhibition was modulated in turn by cAMP interactions with those C-terminal regions. Our data indicate, therefore, that characteristics of anesthetic inhibition of $I_{\mathrm{h}}$ are determined by both HCN subunit composition and prevailing cAMP levels.

\section{Materials and Methods}

Brain slice preparation. Neonatal rats (7-14 d postnatal) were decapitated under ketamine and xylazine anesthesia, and transverse slices $(200 \mu \mathrm{m})$ were prepared from the brainstem and thalamus using a microslicer (DSK 1500E; Dosaka, Kyoto, Japan) in ice-cold substituted Ringer's solution containing the following (in $\mathrm{mM}$ ): 260 sucrose, $3 \mathrm{KCl}, 5 \mathrm{MgCl}_{2}, 1$ $\mathrm{CaCl}_{2}, 1.25 \mathrm{NaH}_{2} \mathrm{PO}_{4}, 26 \mathrm{NaHCO}_{3}, 10$ glucose, and 1 kynurenic acid. Slices were incubated for $1 \mathrm{~h}$ at $37^{\circ} \mathrm{C}$ and subsequently at room temperature in normal Ringer's solution containing the following (in mM): 130 $\mathrm{NaCl}, 3 \mathrm{KCl}, 2 \mathrm{MgCl}_{2}, 2 \mathrm{CaCl}_{2}, 1.25 \mathrm{NaH}_{2} \mathrm{PO}_{4}, 26 \mathrm{NaHCO}_{3}$, and 10 glucose. Both substituted and normal Ringer's solutions were bubbled with $95 \% \mathrm{O}_{2} / 5 \% \mathrm{CO}_{2}$. Slices were submerged in a chamber mounted on a fixed-stage microscope (Axioskop FS; Zeiss, Oberkochen, Germany), and neurons were visualized using Nomarski optics and identified by their location in the slice.

HCN channel constructs. We obtained mouse HCN channel constructs from Drs. B. Santoro and S. A. Siegelbaum (Columbia University, New York, NY). Wild-type mHCN1 and mHCN2 were provided in pTracer and subcloned into pcDNA3 using EcoRI and XbaI. To generate a concatemeric HCN1-HCN2 construct, we used overlap extension PCR to produce a PshAI-NheI fragment that spliced the final leucine of HCN1 directly in frame with the initiating methionine of $\mathrm{HCN} 2$, with no additional intervening sequence. HCN channel chimeras are exactly as described previously (Wang et al., 2001); they were obtained in pGH19 or pGHE and subcloned into pcDNA3-HE3 for recording in HEK293 cells.

To engineer a pair of chimeras that swapped the core channel domains of transmembrane segments between HCN1 and HCN2, we first used the primers 5'-CACCAGTGGGAAGAGATTTTCCACATGACCTATGACCT- $3^{\prime}$ and $5^{\prime}$-TTGTGGAATGACATGTATTGCTCTACTTGCTTGTACTTCTC-3' to amplify a 431 bp cDNA fragment that included the fifth and sixth transmembrane domains and intervening pore helix of $\mathrm{HCN} 1$ or HCN2, respectively. These fragments were then purified and used as primers in a QuickChange PCR to generate HCN1-2(S5-S6) and HCN2-1(S5-S6). We used QuickChange PCR to introduce stop codons immediately after the final transmembrane segment of HCN1(S391stop) or HCN2(S444stop) to make the C-terminal deletion mutants, HCN1 $\Delta$ Cterm and HCN2 $\Delta$ Cterm, and we generated an HCN1(V473stop) and HCN2(V526stop) to delete the cyclic nucleotide binding domain (CNBD) and extreme $\mathrm{C}$ terminus of the two $\mathrm{HCN}$ subunits (HCN1 $\triangle \mathrm{CNBD}$ and HCN2 $\triangle \mathrm{CNBD}$ ). The sequences of all mutated channel constructs were verified in full.

Heterologous expression of HCN channel constructs. HEK293 cells were cultured using standard procedures and transiently transfected with $\mathrm{HCN}$ channel constructs, together with a green fluorescent protein plasmid (pGreenLantern; Invitrogen, San Diego, CA), using either $\mathrm{CaPO}_{4}$ precipitation or Lipofectamine 2000 reagent (Invitrogen). Recordings were obtained 2-4 d after transfection.

Electrophysiology. Whole-cell recordings were obtained at room tem- perature using 3-5 $\mathrm{M} \Omega$ patch pipettes and an Axopatch 200B amplifier in a bath solution composed of the following (in $\mathrm{mm}$ ): $140 \mathrm{NaCl}, 3 \mathrm{KCl}, 2$ $\mathrm{MgCl}_{2}, 2 \mathrm{CaCl}_{2}, 10$ HEPES, and 10 glucose, $\mathrm{pH} 7.3$, that was perfused continuously $(\sim 2 \mathrm{ml} / \mathrm{min})$; the bath solution for slice recordings included tetrodotoxin $(0.75 \mu \mathrm{M})$ and was buffered to $\mathrm{pH} 6.5$ to block $\mathrm{pH}$ and anesthetic-sensitive background $\mathrm{K}^{+}$currents (Sirois et al., 2002); for recording $\mathrm{HCN}$ currents in $\mathrm{HEK} 293$ cells, the $\mathrm{KCl}$ concentration was raised to $25 \mathrm{~mm}$ (substituted equimolar for $\mathrm{NaCl}$ ). Isoproterenol and 3-isobutyl-1-methylxanthine (IBMX) were obtained from Sigma (St. Louis, MO) and added to the bath at 1 and $100 \mu \mathrm{M}$, respectively. Internal solution contained the following (in mM): $120 \mathrm{KCH}_{3} \mathrm{SO}_{3}, 4 \mathrm{NaCl}, 1$ $\mathrm{MgCl}_{2}, 0.5 \mathrm{CaCl}_{2}, 10$ HEPES, 10 EGTA, $3 \mathrm{Mg}$-ATP, and 0.3 GTP-Tris, $\mathrm{pH}$ 7.2; when noted, pipette solution was supplemented with cAMP (0.01-1.0 mM). Bath solutions containing anesthetics were equilibrated by bubbling with a room air gas mixture $\left(21 \% \mathrm{O}_{2} /\right.$ balance $\left.\mathrm{N}_{2}\right)$ directed through calibrated vaporizers (Ohmeda, Liberty Corner, $\mathrm{NJ}$ ); anesthetic concentrations were determined by gas chromatography of perfusate samples.

Data acquisition and analysis. Voltage-clamp commands were applied, and currents were recorded and analyzed using pClamp software and a Digidata 1322A digitizer (Axon Instruments, Union City, CA). Cells were held at -40 to $-50 \mathrm{mV}$ and subjected to a two-pulse protocol, with incrementing hyperpolarizing steps to generate time-dependent hyperpolarization-activated currents $\left(I_{\mathrm{h}}, \mathrm{HCN}\right)$, followed immediately by a step to fixed potential for measurement of tail currents. For $I-V$ curves, current amplitudes during conditioning voltage steps were determined as the difference in current at the end of the voltage step from that measured at the beginning of the step, immediately after the capacitive transient (i.e., time-dependent current); no additional leak subtraction was applied for analysis. The amplitude of time-dependent current at the end of $2-6 \mathrm{~s}$ hyperpolarizing voltage steps (to -120 or $-130 \mathrm{mV}$ ) was taken as maximal available current. Time constants $(\tau)$ were obtained from biexponential fits to current activated during the hyperpolarizing steps. Absolute tail current amplitudes were measured, normalized between the maximum and minimum values, and fitted with Boltzmann curves for derivation of activation parameters $\left(V_{1 / 2}\right.$ and $\left.k\right)$. Control experiments using longer conditioning steps at intermediate voltages $(8-10 \mathrm{~s})$ for the slower HCN2 channels yielded $V_{1 / 2}$ values that were virtually identical to those obtained with steps of $4-6 \mathrm{~s}$ duration. All data are expressed as means \pm SEM; statistical tests included one- and twoway ANOVA, Student's $t$ test, or linear regression, as indicated, with $p<$ 0.05 as criteria for acceptance as statistically significant.

In situ hybridization. In situ hybridization was performed for detection of HCN channel mRNA. Transverse brain sections (fresh frozen, $10 \mu \mathrm{m}$ ) were obtained from Sprague Dawley rats, thaw mounted onto charged slides, and fixed, dehydrated, and delipidated. Sections were hybridized to $\left[{ }^{33} \mathrm{P}\right] \mathrm{UTP}$-labeled cRNA probes transcribed from EcoRI-digested HCN1- and HCN2-pcDNA3 using T7 RNA polymerase (Promega, Madison, WI $)$ in hybridization buffer $\left(50 \times 10^{6} \mathrm{cpm} / \mathrm{ml}\right)$ containing $50 \%$ formamide, $4 \times$ SSC $(1 \times$ SSC: $150 \mathrm{~mm} \mathrm{NaCl}, 15 \mathrm{~mm}$ sodium citrate, $\mathrm{pH}$ 7 ), $1 \times$ Denhardt's solutions ( $0.02 \%$ each of Ficoll, polyvinylpyrrolidone, and bovine serum albumin), 10\% dextran sulfate, $100 \mathrm{~mm}$ DTT, 250 $\mu \mathrm{g} / \mathrm{ml}$ yeast tRNA, and $0.5 \mathrm{mg} / \mathrm{ml}$ salmon testes DNA. After hybridization, sections were rinsed in SSC and treated with RNase A $[0.1 \mathrm{mg} / \mathrm{ml}$ in $10 \mathrm{~mm}$ Tris, $500 \mathrm{~mm} \mathrm{NaCl}$, and $1 \mathrm{~mm}$ EDTA, $\mathrm{pH} \mathrm{7,} \mathrm{for} 30 \mathrm{~min}$ at $37^{\circ} \mathrm{C}$ (Boehringer Mannheim, Mannheim, Germany)] and exposed to film (Hyperfilm $\beta$ MAX; Amersham Biosciences, Arlington Heights, IL) for $4 \mathrm{~d}$ or to liquid emulsion (NTB-2; Eastman Kodak, Rochester, NY) for $3-5$ weeks.

\section{Results}

Halothane inhibits native motoneuronal $I_{\mathrm{h}}$ by shifting voltage dependence and decreasing peak current amplitude

Although $I_{\mathrm{h}}$ is best known for contributions to pacemaker activity, the current can also affect integrative properties in nonpacemaker cells (for review, see Pape, 1996). In motoneurons, which are not themselves pacemakers, $I_{\mathrm{h}}$ is a prominent compo- 
A

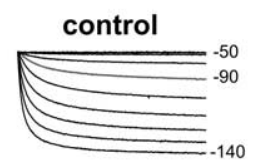

tail currents at $-90 \mathrm{mV}$

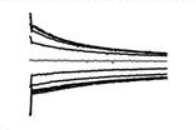

C

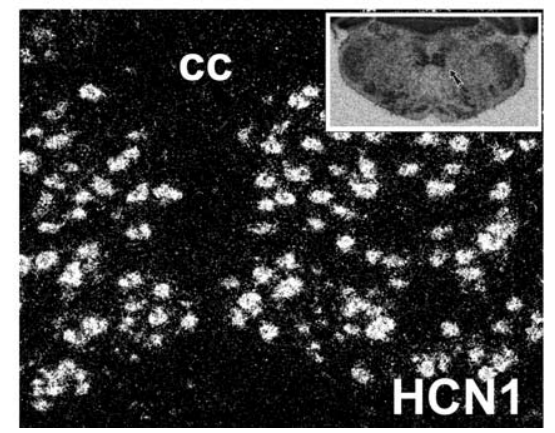

B
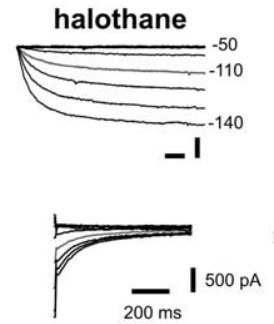
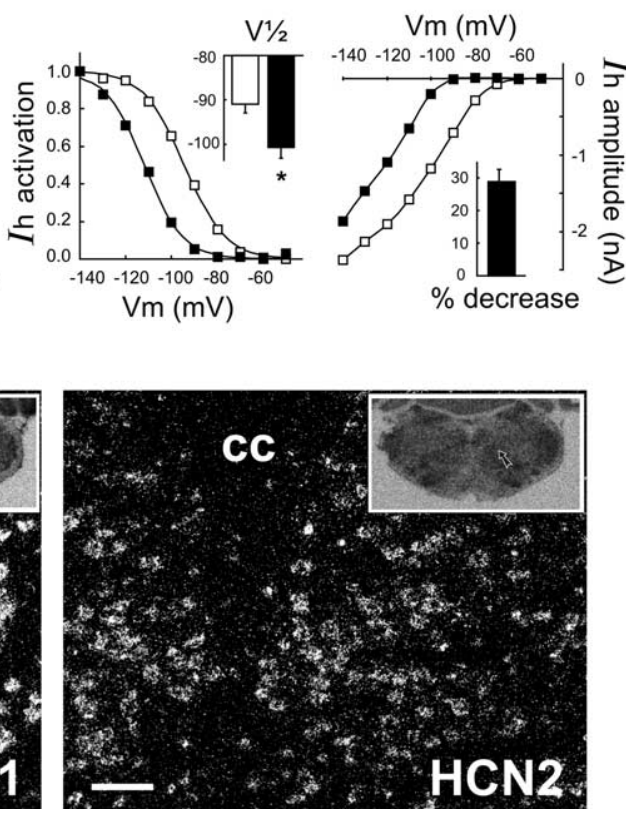

Figure 1. Halothane causes a hyperpolarizing shift in $/ \mathrm{h}$ activation and a decrease in maximal $/ \mathrm{h}$ amplitude in motoneurons that express $\mathrm{HCN} 1$ and $\mathrm{HCN} 2$ subunits. $\boldsymbol{A}$, Representative sample currents from motoneurons during hyperpolarizing steps $(\Delta-10 \mathrm{mV})$ from -50 to $-140 \mathrm{mV}$ (top), before and during exposure to halothane $(0.8 \mathrm{~mm})$. Tail currents were evoked at $-90 \mathrm{mV}$ after the hyperpolarizing step (bottom); traces near the half-activation potential $\left(V_{1 / 2}\right)$ under control and halothane conditions are shaded. For presentation of motoneuronal data, leak currents were subtracted from sample records obtained during the initial hyperpolarizing voltage steps (top). $\boldsymbol{B}$, Left, Activation curves for $I_{\mathrm{h}}$ were determined from Boltzmann fits to normalized tail currents; averaged data ( \pm SEM; inset) indicate that halothane induced an approximate $-10 \mathrm{mV}$ shift in $V_{1 / 2}$ of activation (from $-90.9 \pm$ 2.0 to $-100.7 \pm 2.4 \mathrm{mV} ; n=9 ; p<0.0001)$. Right, Halothane decreased current amplitude measured at the end of hyperpolarizing steps; averaged data ( \pm SEM; inset) show that halothane decreased motoneuronal $I_{\mathrm{h}}$ at $-130 \mathrm{mV}$ by $28.8 \pm 3.8 \%(n=$ $9 ; p<0.001)$. C, In situ hybridization with $\left[{ }^{33} \mathrm{P}\right]$-labeled CRNA probes revealed high levels of HCN1 and moderate levels of HCN2 subunit transcripts in motoneurons of the hypoglossal nucleus. Dark-field images of emulsion-dipped slides illustrate the localization of HCN1 and HCN2 mRNA in hypoglossal motoneurons. Scale bar, $50 \mu \mathrm{m}$. Film autoradiographs (insets) depict representative brainstem sections. cc, Central canal.

nent of subthreshold current (Rekling et al., 2000). As shown for a representative cell in Figure 1, the inhalational anesthetic halothane caused a robust inhibition of motoneuronal $I_{\mathrm{h}}$, an effect that was manifest in at least two ways: analysis of tail currents at $-90 \mathrm{mV}$ after hyperpolarizing voltage steps to different membrane potentials revealed a halothane-induced leftward shift in the voltage range of activation (Fig. $1 A, B$, left), whereas comparison of steady-state currents obtained at the end of the hyperpolarizing steps indicated that halothane decreased maximal $I_{\mathrm{h}}$ amplitude (Fig. $1 A, B$, right). On average, $0.8 \mathrm{~mm}$ halothane caused an approximate $-10 \mathrm{mV}$ shift in $V_{1 / 2}$, the half-activation voltage, while also producing $\sim 29 \%$ decrease in current amplitude measured at $-130 \mathrm{mV}$. Although halothane appeared to slow $I_{\mathrm{h}}$ activation kinetics at all potentials, there was no difference in activation kinetics when membrane potential was corrected for the hyperpolarizing shift in $V_{1 / 2}$ of activation (i.e., $V_{\mathrm{m}}-V_{1 / 2}$ ) (see supplemental data, available at www.jneurosci.org as supplemental material). Thus, halothane-induced inhibition of $I_{\mathrm{h}}$ in motoneurons results from a shift in activation to more hyperpolarized potentials and a decrease in maximal current amplitude, with no change in the relationship between activation kinetics and activation voltage. It is important to point out that the combined effect of this inhibition is essentially a complete abolition of $I_{\mathrm{h}}$ at all membrane potentials depolarized to $-90 \mathrm{mV}$ (e.g., see Fig. $1 B$, right, filled squares).
Motoneurons express HCN1 and HCN2 subunits, which are differentially modulated by anesthetics

The HCN family of ion channels represents the molecular substrate for neuronal $I_{\mathrm{h}}$, and in situ hybridization experiments indicate that motoneurons express two HCN subunits, HCN1 and HCN2 (Fig. $1 C$ ), with an apparent predominance of the HCN1 subunit (Moosmang et al., 1999; Monteggia et al., 2000; Santoro et al., 2000). These two HCN subunits produce homomeric channels that differ markedly in activation properties; HCN2 currents activate more slowly and at more hyperpolarized potentials than $\mathrm{HCN} 1$ currents (Biel et al., 1999; Santoro and Tibbs, 1999; Kaupp and Seifert, 2001).

We expressed these cloned HCN subunits as homomeric channels in HEK293 cells and found that they also differed in their modulation by halothane (Fig. 2). Specifically, the two anesthetic effects on $I_{\mathrm{h}}$ in motoneurons (hyperpolarizing shift in $I_{\mathrm{h}}$ activation and decrease in maximal $I_{\mathrm{h}}$ amplitude) segregated with the two individual subunits. Thus, halothane caused a hyperpolarizing shift in $V_{1 / 2}$ in cells expressing $\mathrm{HCN} 1$, with little effect on amplitude (Fig. $2 A, B$, top panels), whereas it decreased $\mathrm{HCN} 2$ current amplitude without substantially changing the voltage range of HCN2 activation (Fig. 2A, $B$, middle panels). Note that the initial $V_{1 / 2}$ of HCN1 $(-80.5 \pm 0.6 \mathrm{mV} ; n=50)$ was substantially more depolarized than that of $\mathrm{HCN} 2$ $(-98.6 \pm 0.6 \mathrm{mV} ; n=57)$, as expected for these cloned channels (Biel et al., 1999; Santoro and Tibbs, 1999; Kaupp and Seifert, 2001).

It is now clear that HCN subunits can form heteromeric channels (Chen et al., 2001; Ulens and Tytgat, 2001; Much et al., 2003). To test effects of halothane on heteromeric $\mathrm{HCN}$ channels, we linked $\mathrm{HCN} 1$ and $\mathrm{HCN} 2 \mathrm{cDNAs}$ in a concatemeric arrangement and expressed the linked construct in HEK293 cells; this HCN1HCN2 heteromeric channel produced hyperpolarizationactivated currents with kinetic and voltage-dependent properties intermediate to those of the constituent HCN1 and HCN2 subunits, as reported previously (Chen et al., 2001; Ulens and Tytgat, 2001). Consistent with an independent contribution of each subunit to halothane inhibition of the heteromeric channel, the anesthetic modulated HCN1-HCN2 currents via mechanisms attributable to both $\mathrm{HCN} 1$ and $\mathrm{HCN} 2$, causing a shift in $V_{1 / 2}$ and a decrease in maximal current amplitude (Fig. $2 A, B$, bottom panels). Indeed, results with heteromeric $\mathrm{HCN} 1-\mathrm{HCN} 2$ channels were most similar to those from motoneurons (compare Figs. $1 B$, $2 B$, bottom panels), because halothane induced an approximate $-10 \mathrm{mV}$ shift in $V_{1 / 2}$ (from $-86.9 \pm 1.2$ to $-96.5 \pm 1.1 \mathrm{mV} ; n=$ 12 ) and $\sim 18 \%$ decrease in maximal HCN1-HCN2 current amplitude.

Halothane also modulated current kinetics in an HCN subunit-dependent manner. When corrected for shifts in $V_{1 / 2}$, halothane caused a speeding of $\mathrm{HCN} 1$ and a slowing of $\mathrm{HCN} 2$ current activation (see supplemental data, available at www. 
jneurosci.org as supplemental material). It is important to note that, although activation kinetics were altered by halothane, changes in activation $\tau$ cannot account for inhibitory actions of the anesthetic on current amplitude or $V_{1 / 2}$ in $\mathrm{HCN}$ channels. The decrease in HCN2 current amplitude by halothane was obtained at the end of $4 \mathrm{~s}$ pulses to $-120 \mathrm{mV}$, at a time point that represents approximately six times the activation $\tau$ at that potential in halothane $(635 \pm 150 \mathrm{~ms} ; n=7)$. Moreover, the inhibition determined by using $6 \mathrm{~s}$ pulses $(28.7 \pm 1.1 \%$ inhibition; $n=3)$, after $\sim 9$ $\tau$, was essentially identical to that obtained with our standard $4 \mathrm{~s}$ pulses $(33.2 \pm 3.3 \%$ inhibition; $n=17)$. Measured values of $V_{1 / 2}$ for $I_{\mathrm{h}}$ can be exaggerated in the hyperpolarized direction under conditions when equilibration is incomplete at intermediate voltages (Kaupp and Seifert, 2001), and this can be particularly problematic if current activation is slow (as it is with HCN2 and especially with HCN2 in halothane). However, our values for $V_{1 / 2}$ with HCN2 were stable with longer conditioning pulse durations $(8-10 \mathrm{~s}$; data not shown). Moreover, we find that halothane had little effect on $V_{1 / 2}$ of the HCN2 channels despite slowing current activation, whereas the anesthetic induced a substantial hyperpolarizing shift in $V_{1 / 2}$ of the HCN1 channels in which it accelerated current activation. Therefore, effects of halothane on $V_{1 / 2}$ of HCN1 and HCN2 are opposite of that expected if they had resulted from altered activation kinetics.

In contrast to its effects on activation kinetics in HCN1 and HCN2 homomeric channels, halothane did not change activation kinetics in HCN1-HCN2 heteromeric channels (see supplemental data, available at www.jneurosci.org as supplemental material). Thus, effects of halothane on HCN1-HCN2 channels were intermediate between those on HCN1 and HCN2 homomeric channels and again resembled more closely the characteristics of inhibition in motoneurons in which halothane did not change current activation kinetics. Together, these data indicate that both HCN1 and HCN2 channel subunits contribute to the motoneuronal $I_{\mathrm{h}}$ current and its inhibition by halothane.

\section{HCN channels are modulated by inhalation anesthetics at clinically relevant concentrations}

Distinct anesthetic actions occur over a submillimolar concentration range, with an $\mathrm{EC}_{50}$ for immobilization of $\sim 0.3 \mathrm{~mm}$ and progressive EEG depression up to $1 \mathrm{mM}$ (Franks and Lieb, 1994; Eckenhoff and Johansson, 1999). Effects of halothane on HCN channels were dose dependent within this clinically relevant concentration range (Fig. $2 C, D$ ). Significant shifts in $V_{1 / 2}$ were evident at $0.3 \mathrm{~mm}$ halothane $(\mathrm{HCN} 1,-9.0 \pm 0.7 \mathrm{mV}, n=26$;
B
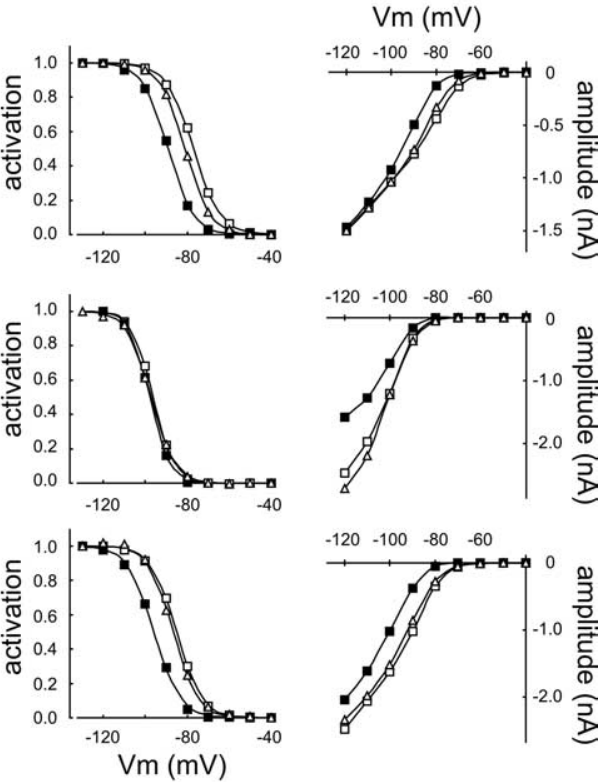

D

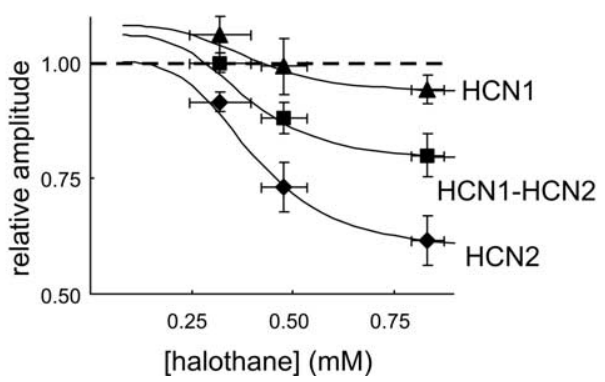

Figure 2. HCN1 and HCN2 subunits are associated, respectively, with a halothane-induced hyperpolarizing shift in $V_{1 / 2}$ or a decrease in maximal current amplitude. $\boldsymbol{A}$, Sample currents from HEK293 cells expressing HCN1, HCN2, or a linked heteromeric HCN1-HCN2 construct evoked by hyperpolarizing voltage steps $(\Delta-10 \mathrm{mV})$ from -40 to $-120 \mathrm{mV}$ before, during, and after ) and followed by a step to $-90 \mathrm{mV}$ for tail current analysis. Shaded traces are near the control $V_{1 / 2}$ and at the same potential in hal othane. $\boldsymbol{B}$, Activation curves were determined from tail currents (left) and steady-state $I-V$ curves from currents at the end of (open triangles) for HCN1, HCN2, or a linked HCN1-HCN2 construct. C, Concentration-response curves for the halothane-induced shift in $V_{1 / 2}$; averaged $\Delta V_{1 / 2}$ values ( $n>8$ per group) were fitted with logistic curves using maximal $\Delta V_{1 / 2}$ of $-16,-4$, and analysis by two-way ANOVA revealed significant main effects for $\mathrm{HCN}$ constructs $\left(F_{(2,146)}=53.5 ; p<0.0001\right)$ and halothane concentrations $\left(F_{(2,146)}=5.7 ; p<0.005\right)$. D , Concentration-response curves for the halothane-induced inhibition of steadyand $\mathrm{EC}_{50}$ values of $0.40,0.40$, and $0.39 \mathrm{~mm}$ for $\mathrm{HCN} 1$ (triangles), HCN2 (diamonds), and HCN1-HCN2 (squares). Data analysis by two-way ANOVA revealed significant main effects for $\mathrm{HCN}$ constructs $\left(F_{(2,142)}=23.3 ; p<0.0001\right)$ and halothane concentrations $\left(F_{(2,142)}=11.1 ; p<0.0001\right)$.

$\mathrm{HCN} 2,-3.6 \pm 0.6 \mathrm{mV}, n=31 ; \mathrm{HCN} 1-\mathrm{HCN} 2,-8.2 \pm 0.7 \mathrm{mV}$, $n=15$ ), with near-maximal shifts in gating obtained at $0.5 \mathrm{~mm}$ halothane for HCN1 (approximately $-13 \mathrm{mV}$ ) and HCN1HCN2 (approximately $-10 \mathrm{mV}$ ). Inhibition of current amplitude was significant at halothane concentrations of $0.5 \mathrm{~mm}$ for both HCN2 ( 27\% inhibition) and HCN1-HCN2 $(\sim 14 \%$ inhibition).

The halogenated ether anesthetic isoflurane had effects essentially identical to those of halothane (data not shown): isoflurane $(0.9 \mathrm{~mm})$ caused a hyperpolarizing shift in $V_{1 / 2}$ of HCN1 and HCN1-HCN2 channels $(-10.1 \pm 0.9 \mathrm{mV}, n=4 ;-10.6 \pm 1.8$ $\mathrm{mV}, n=6)$, with little effect on $\mathrm{HCN} 2(-3.7 \pm 1.5 \mathrm{mV} ; n=6)$, 
A

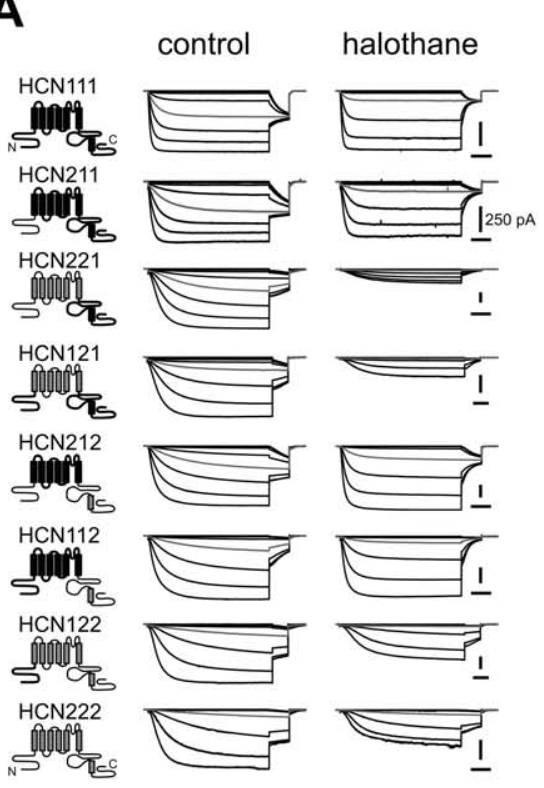

C

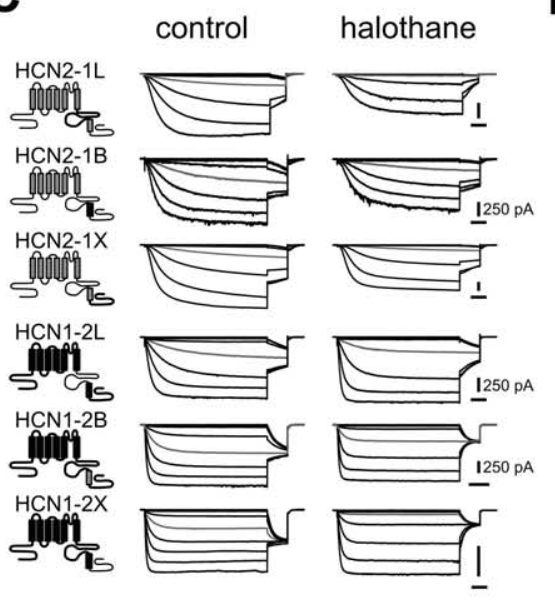

E

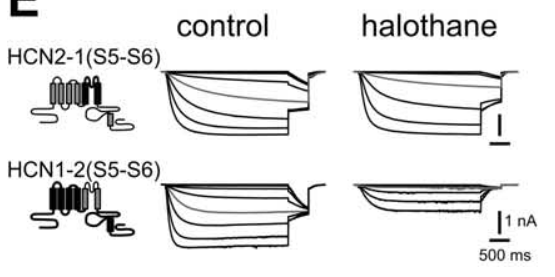

B

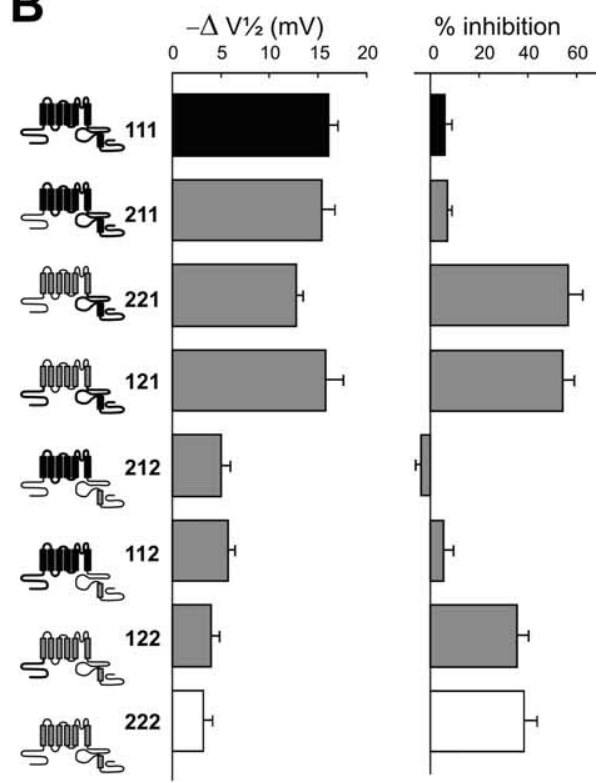

D

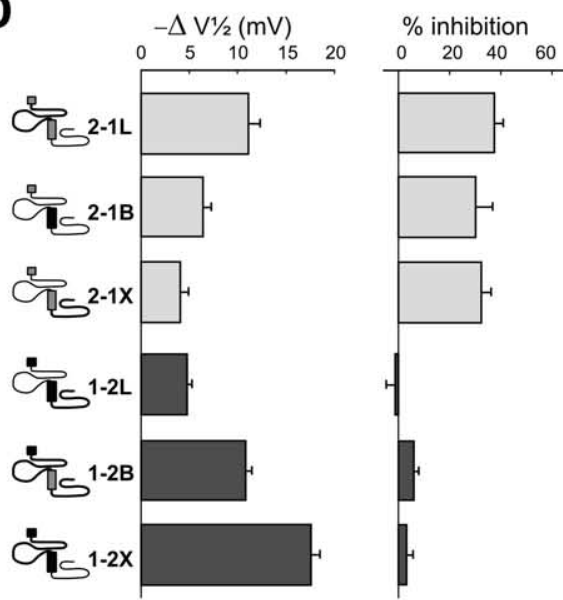

$\mathbf{F}$

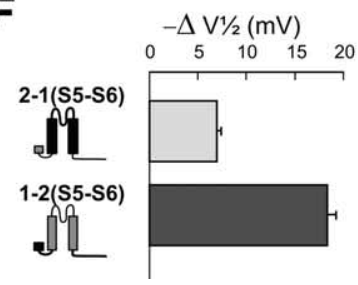

In chimeric channels, distinct channel domains are necessary for $\mathrm{HCN}$ gating and amplitude modulation by halothane Anesthetic actions on current amplitude and gating segregated with individual HCN subunits, suggesting that separate structural determinants might account for these distinct anesthetic effects. We therefore tested effects of halothane on chimeric constructs in which the $\mathrm{N}$ terminus, transmembrane segments, or $\mathrm{C}$ terminus were exchanged between $\mathrm{HCN} 1$ and $\mathrm{HCN} 2$ subunits [denoted by HCN: $\mathrm{N}$ terminus, transmembrane segments, $\mathrm{C}$ terminus (as by Wang et al., 2001)]. As illustrated in Figure 3, $A$ and $B$ (also see supplemental data, available at www.jneurosci.org as supplemental material), halothane caused a large hyperpolarizing shift in $V_{1 / 2}$ only in those chimeric channels that contained the $\mathrm{C}$ terminus of $\mathrm{HCN} 1$, whereas inhibition of current amplitude by halothane tracked with the transmembrane segments of HCN2. In two constructs containing the transmembrane segments of $\mathrm{HCN} 2$ and the $\mathrm{C}$ terminus of HCN1 (HCN221 and HCN121), halothane caused both a shift in $V_{1 / 2}$ and a decrease in current amplitude. Conversely, halothane had little effect on gating or current amplitude in HCN212 and $\mathrm{HCN} 112$ chimeric channels that contained the $\mathrm{C}$ terminus of $\mathrm{HCN} 2$ and the transmembrane segments of HCN1. Halothane was not completely without effect on these latter two chimeras, because it still caused a speeding of current activation (Fig. 3A, sample traces). In fact, the enhancement of activation kinetics typically associated with anesthetic modulation of $\mathrm{HCN} 1$ was retained in chimeras containing HCN1 transmembrane segments, whereas the slowed activation typical of HCN2 was observed in chimeras containing $\mathrm{HCN} 2$ transmembrane segments (data not shown).

Additional chimeric constructs were used to localize more precisely the effects of halothane on gating and current amplitude within the $\mathrm{C}$ terminus and the transmembrane segments, respectively. The $\mathrm{C}$ terminus was divided into three regions (denoted $\mathrm{X}, \mathrm{B}$, and $\mathrm{L}$ ): an extreme $\mathrm{C}$ terminal region, the CNBD, and a proximal C-linker region (i.e., the sequence between the final transmembrane spanning domain and the CNBD). Swapping these individual domains in chimeric channels revealed that halothane-induced actions on $V_{1 / 2}$ of activation depend on both the whereas it inhibited maximal current amplitude in HCN2 and HCN1-HCN2 channels (19.6 $\pm 4 \%$ inhibition, $n=6$; $20.6 \pm$ $7.2 \%$ inhibition, $n=6$ ) but did not decrease HCN1 current amplitude $(-3.7 \pm 4.1 \%$ inhibition; $n=4)$.
$\mathrm{CNBD}$ and the $\mathrm{C}$ linker of the subunits, with the $\mathrm{C}$-linker domain appearing most critical (Fig. 3C,D). Thus, although incorporating either the HCN1 linker domain or CNBD into an HCN2 subunit (HCN2-1L, HCN2-1B) enhanced the halothane- 
induced hyperpolarizing shift in $V_{1 / 2}$, the anesthetic effect on gating was fully lost only when the linker domain of HCN2 was included in HCN1 (HCN1-2L). As expected based on chimeras described above, amplitude inhibition by halothane was prominent in all constructs that included the transmembrane segments of HCN2 but was absent in all constructs containing an HCN1 transmembrane region (see Fig. $3 D$, right).

We produced a final pair of chimeras that swapped the core channel domains of the transmembrane segments (i.e., the fifth and sixth transmembrane domains and the intervening pore helix, S5-S6). As shown in Figure 3, E and F, current amplitude was not inhibited by halothane in the HCN2 channel construct that included pore-forming regions from $\mathrm{HCN} 1$ [i.e., HCN1-2(S5-S6), also HCN1-2P], whereas incorporating the corresponding channel core of HCN2 into HCN1 [i.e., HCN2-1(S5-S6), also HCN2-1P] conferred amplitude modulation by halothane, together with a shift in gating. It is worth noting that effects of halothane on current kinetics also localized to this S5-S6 core channel region; as with HCN2, halothane slowed current activation in $\mathrm{HCN1}-$ 2(S5-S6) constructs, and, as with HCN1, halothane sped activation in the HCN21(S5-S6) construct (data not shown).

In summary, data from these experiments with chimeric constructs indicate that halothane inhibits channels containing S5-S6 domains of HCN2 by decreasing current amplitude and activation kinetics; conversely, halothane causes a shift in $V_{1 / 2}$ of activation in channels containing HCN1 $\mathrm{C}$-terminal regions, especially the $\mathrm{C}$-linker domain. These results are consistent with an interpretation that independent inhibitory actions of halothane (on amplitude and $V_{1 / 2}$ ) are mediated at distinct sites within the channel. However, because different domains from HCN1 and HCN2 subunits can allosterically modulate basal channel function in distinct ways (Wainger et al., 2001; Wang et al., 2001; Zagotta et al., 2003; Craven and Zagotta, 2004), it is also possible that the form of anesthetic inhibition is actually determined by the basal properties of the channel that those domains regulate (see below). We explored this possibility by taking advantage of the ability of cAMP to abrogate differences in basal inhibition between HCN1 and HCN2 channels.

\section{Gating and amplitude inhibition by halothane depend primarily on basal inhibition imposed by the $\mathrm{C}$ terminus on HCN channels}

A well supported model for HCN channel modulation by cAMP posits that the C-terminal CNBD provides a tonic allosteric inhibition of channel gating that is transmitted to the channel "gate" via the intervening C-linker domain; binding of cAMP to the $\mathrm{CNBD}$ relieves the tonic inhibition, thereby allowing the channel to open at more depolarized potentials (Wainger et al., 2001; Wang et al., 2001; Zagotta et al., 2003; Craven and Zagotta, 2004). According to this model, the gating propensity of the core chan- nel domains of HCN1 and HCN2 are approximately equivalent in the absence of their respective $\mathrm{C}$ terminal domains [i.e., $\mathrm{C}$ terminal deletion mutants have very similar $V_{1 / 2}$ of activation (Wainger et al., 2001)], but allosteric inhibition is more prominent in channels containing an HCN2 C terminus than in those with an HCN1 C terminus. The different inhibitory effects of the respective proximal $\mathrm{C}$-terminal domains are thus proposed to account for the more hyperpolarized initial $V_{1 / 2}$ in HCN2 than in HCN1 channels and for the larger depolarizing shift in $V_{1 / 2}$ by cAMP in HCN2 channels (i.e., stronger relief by cAMP of the basal inhibition) (Wainger et al., 2001; Wang et al., 2001).

Strikingly, our chimera experiments map differences in halothane-induced modulation of gating of HCN1 and HCN2 to the same proximal C-terminal channel domains responsible for differences in effects of cAMP on these channels (Wainger et al., 2001; Wang et al., 2001). In the case of halothane, however, the shift in $V_{1 / 2}$ is in the opposite direction as that induced by cAMP and with an opposite subunit sensitivity (i.e., HCN1 is more sensitive than HCN2). To confirm this, we recorded HCN channels and chimeric constructs in the presence of saturating concentrations of cAMP in the pipette $(50 \mu \mathrm{M})$. As shown in Figure $4 A$ and in agreement with previous work (Wang et al., 2001), we found that cAMP caused a depolarizing shift in $V_{1 / 2}$ in chimeric channels that, although influenced by transmembrane segments, was primarily determined by C-terminal domains. Notably, the pattern of modulation by cAMP of these chimeric HCN channels is 
A

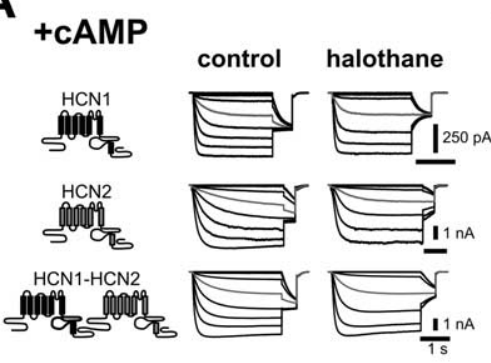

D

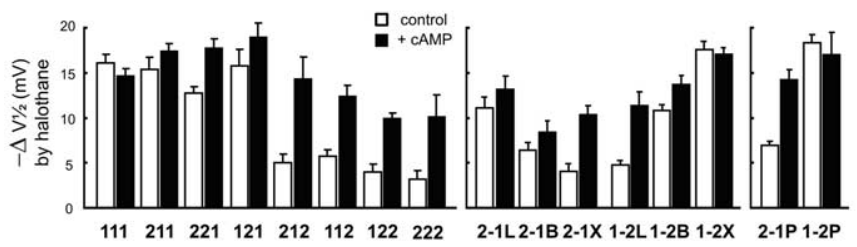

F

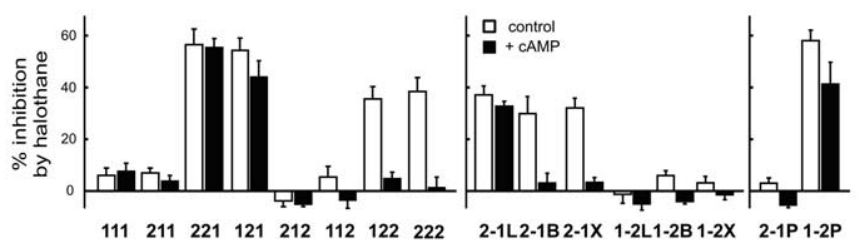

B

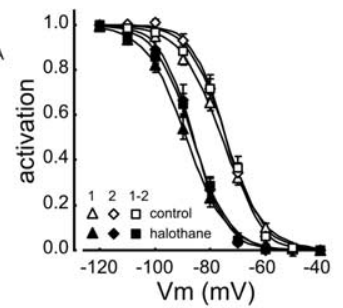

E

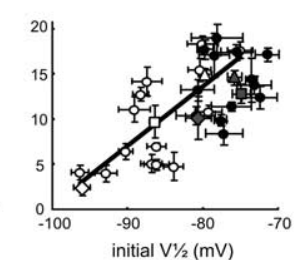

G

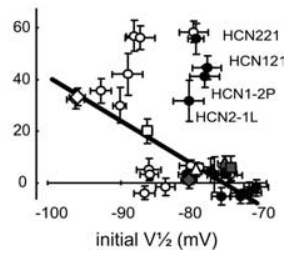

Figure 5. Relief of basal inhibition by CAMP changes the characteristics of halothane inhibition in channels that include an HCN2 C terminus. HCN channel currents were recorded in HEK293 cells with pipettes that contained saturating concentrations of CAMP (50 $\mu \mathrm{M})$. A, Sample currents evoked from cells expressing HCN1, HCN2, or heteromeric HCN1-HCN2 channels ( $n>6$ per group), before and during exposure to a maximal concentration of halothane $(0.8 \mathrm{~mm})$; shaded traces are near the control $V_{1 / 2}$ and at the same potential in halothane. Note that halothane had little effect on current amplitude from cAMP-loaded cells expressing HCN channels. $\boldsymbol{B}$, In the presence of CAMP, averaged activation curves ( \pm SEM) were determined from tail currents under control conditions and during exposure to halothane for HCN1, HCN2, and HCN1-HCN2. C, Relationship between initial $V_{1 / 2}$, a measure of basal inhibition, and either the hyperpolarizing shift in $V_{1 / 2}$ (top) or the amplitude inhibition (bottom) induced by halothane in cells expressing HCN1, HCN2, and HCN1-HCN2 under control conditions or in the presence of CAMP. D, Averaged shift in $V_{1 / 2}$ ( \pm SEM) for all chimeric constructs determined in the absence and presence of CAMP. $\boldsymbol{E}$, Relationship between initial $V_{1 / 2}$ and the hyperpolarizing shift in $V_{1 / 2}$ induced by halothane for all HCN channel constructs recorded in the absence and presence of cAMP; the overlaid regression line is from Figure $4 B$. In the presence of $C A M P$, halothane caused a voltage-dependent shift in gating in all HCN constructs. $F$, Averaged amplitude inhibition ( \pm SEM) for HCN channel constructs determined in the absence and presence of CAMP. G, Relationship between initial $V_{1 / 2}$ and current inhibition by halothane for all HCN channel constructs recorded in the absence and presence of CAMP; the overlaid line is the fit obtained to data from HCN1, HCN2, and HCN1-HCN2 channels. In the presence of CAMP, amplitude inhibition was lost from all HCN constructs except those indicated, which included an HCN1 C terminus together with an $\mathrm{HCN} 2$ channel core. Data points represent averaged data ( \pm SEM; $n>6$ per construct). In $\boldsymbol{E}$ and $\mathbf{G}$, data are from HCN1 (triangles), HCN2 (diamonds), HCN1-HCN2 (squares), and chimeric HCN channels (circles) in the absence (open symbols) and presence (filled symbols) of cAMP.

opposite that described above for halothane (compare Fig. $4 \mathrm{~A}$ with Fig. 3).

In light of the allosteric inhibition model for cAMP modulation, we examined the correlation between initial $V_{1 / 2}$ of the HCN channel constructs and either the hyperpolarizing shift caused by halothane (Fig. $4 \mathrm{~B}$, left) or the depolarizing shift in $V_{1 / 2}$ caused by cAMP (Fig. $4 B$, right): HCN constructs with more hyperpolarized initial $V_{1 / 2}$ show relatively smaller hyperpolarizing shifts by halothane and larger depolarizing shifts by cAMP, and, conversely, constructs with more depolarized initial $V_{1 / 2}$ have larger shifts by halothane and smaller shifts by cAMP. Indeed, the negative correlation between the depolarizing shift in $V_{1 / 2}$ caused by cAMP and the hyperpolarizing shift in $V_{1 / 2}$ induced by halothane was especially tight across all of these constructs (Fig. $4 C$ ), with a slope that approaches $-1(-0.9)$. Thus, mutations that change the initial $V_{1 / 2}$ of the HCN channels have nearly equal but oppo- site effects on the modulation by cAMP and halothane. Interpreted in terms of the model described above for differences in cAMP modulation of HCN channels, this suggests that subunit-specific differences in effects of halothane on gating might reflect the degree of tonic allosteric inhibition associated with each chimeric subunit; that is, channels with less basal inhibition imposed by the C-terminal domains are more sensitive to inhibitory gating effects of halothane.

To test directly the hypothesis that differences in effects of halothane reflect the degree of basal inhibition associated with each subunit, we examined halothane modulation of HCN channel constructs after relieving that basal inhibition with cAMP. Sample currents from homomeric $\mathrm{HCN} 1$ and HCN2 and heteromeric HCN1-HCN2 channels recorded with cAMP demonstrate that halothane had little effect on maximal current amplitude in any construct (Fig. 5A). Moreover, as presented in Figure $5 B$, the initial $V_{1 / 2}$ was relatively depolarized and essentially identical for all these constructs in the presence of cAMP (approximately $-75 \mathrm{mV}$ ), and, under these conditions of reduced basal inhibition, the hyperpolarizing shift induced by halothane was also similar.

The most dramatic differences in halothane modulation attributable to cAMP were observed with HCN2 channel currents, for which halothane induced a substantial shift in $V_{1 / 2}$ (approximately -10 $\mathrm{mV}$ ) and inhibited current amplitude by $<10 \%$ in the presence of cAMP, actions that were clearly different from the more modest shift and much greater amplitude inhibition $(\sim 40 \%)$ induced by halothane when cAMP was not included in the pipette. We obtained similar results when cAMP levels were increased by forskolin (10 $\mu \mathrm{M}$, bath applied); halothane decreased HCN2 amplitude by $<10 \%(8.9 \pm$ $5.1 \%$ inhibition; $n=11$ ) while inducing an approximate $-10 \mathrm{mV}$ shift in $V_{1 / 2}(-10.1 \pm 2.7 \mathrm{mV} ; n=11)$. The relationship between basal inhibition (i.e., the initial $V_{1 / 2}$ before a halothane challenge) and either the halothane-induced shift in $V_{1 / 2}$ (top) or amplitude inhibition (bottom) is plotted for HCN1, HCN2, and HCN1-HCN2, with and without cAMP, in Figure 5C. Overall, cAMP had little effect on halothane inhibition of HCN1 currents (triangles), consistent with its relatively minor modulation of the basal inhibition of those channels. Conversely, cAMP clearly relieved basal inhibition (i.e., caused a depolarizing shift in initial $V_{1 / 2}$ ) in HCN2 channels (diamonds), and to a lesser extent in HCN1-HCN2 heteromeric channels (squares), and correspondingly enhanced the gating effects of halothane while decreasing its inhibition of current amplitude.

We also determined the effect of cAMP on halothane-induced inhibition in all chimeric HCN channels (Fig. 5D-G). When recorded with cAMP in the pipette, the initial $V_{1 / 2}$ was relatively 
depolarized for all constructs, and halothane caused a large hyperpolarizing shift in $V_{1 / 2}$ in all mutant channels. This included those chimeras containing a C-linker domain from $\mathrm{HCN} 2$ in which halothane induced only a small shift in $V_{1 / 2}$ under control conditions but an augmented shift when cAMP was included in the pipette (e.g., see Fig. 5D, HCN212, HCN1-2L, or HCN2-1P). As shown in Figure $5 E$, the relationship between initial $V_{1 / 2}$ and the anesthetic-induced shift in $V_{1 / 2}$ for all chimeric channels conformed to that described above for wild-type channels. Also, for chimeric channels that included an HCN2 C-linker domain, if halothane had a strong effect on current amplitude in control conditions, the amplitude modulation was lost in the presence of cAMP (e.g., see Fig. 5F, HCN122, HCN2-1B or HCN2-1X). Note, however, that amplitude inhibition was retained in chimeras in which an $\mathrm{HCN} 2$ core was combined with an HCN1 C linker (constructs indicated in Fig. 5G). This may reflect differences in conformational coupling between modules from different subunits in these chimeric channels (Craven and Zagotta, 2004) because the HCN1 C linker is less effective in conveying cAMP modulation to HCN2 core domains (Wang et al., 2001).

So, cAMP had little effect on the form of anesthetic modulation in weakly cAMP-sensitive HCN channels (i.e., HCN1 and chimeras containing an $\mathrm{HCN} 1$ proximal $\mathrm{C}$ terminus). In contrast, for the most strongly cAMP-sensitive HCN channels (i.e., $\mathrm{HCN} 2$ or chimeras containing an HCN2 core together with the proximal $\mathrm{C}$ terminus of $\mathrm{HCN} 2$ ), cAMP reduced basal inhibition and converted halothane inhibition from primarily a decrease in maximal current amplitude to a more HCN1-like pattern (i.e., a hyperpolarizing shift in $V_{1 / 2}$ with little amplitude modulation).

\section{Gating and amplitude inhibition by halothane are preserved in C-terminal deletion mutants of $\mathrm{HCN}$ channels}

The data indicate that the form of channel inhibition by halothane (gating shift or amplitude inhibition) depends to a large extent on the C-terminal domains of the channels, particularly the $\mathrm{C}$ linker, and the extent to which those domains modulate basal channel activity. We wondered whether the primary site of halothane action was actually contained in those C-terminal regions, or whether those domains provide an allosteric modulation of halothane effects that are mediated elsewhere in the channel. To test this, we examined inhibition by halothane of $\mathrm{HCN}$ channel mutants in which the $\mathrm{C}$ terminus was deleted beyond the C linker in both HCN1 and HCN2 (i.e., HCN1 $\Delta$ CNBD and $\mathrm{HCN} 2 \Delta \mathrm{CNBD}$ ) or removed completely by truncation immediately after the final transmembrane segment of $\mathrm{HCN} 1$ (HCN1 $\Delta$ Cterm); we could not test the corresponding complete C-terminal deletion in HCN2 because, as reported previously (Wainger et al., 2001), we found that HCN2 $\Delta$ Cterm did not generate currents. As shown in the sample records and activation plots of Figure 6, halothane caused a strong shift in $V_{1 / 2}$ together with a robust inhibition of maximal current amplitude in all deletion mutants. These data indicate that neither inhibitory action of halothane requires a direct interaction of the anesthetic
B
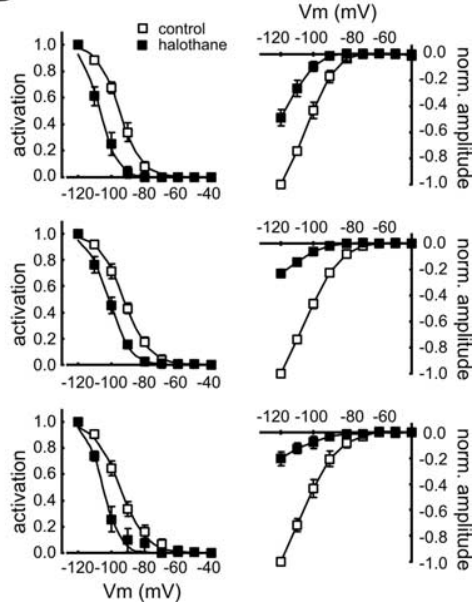

Figure 6. In C-terminally deleted HCN channels, halothane inhibition includes both a decrease in current amplitude and a shift $\left.\right|^{1500 \mathrm{pA}}$ transmembrane segment (HCN1 $\triangle$ Cterm) or after the C linker (HCN1 $\triangle C N B D$ and $H C N 2 \triangle C N B D)$. A Sample currents from the under control conditions (open squares) and during exposure to halothane (filled squares). Halothane induced a shift in $V_{1 / 2}$ of activation and a decrease in maximal current amplitude.

with the C-terminal domains, and that inhibition by halothane is essentially identical in HCN1 and HCN2 subunits in the absence of their respective $\mathrm{C}$ termini. Together with previous results from chimeric channels, these data also imply that proximal C-terminal regions modulate, rather than mediate, HCN channel inhibition by halothane.

\section{Inhibition by halothane also depends on prevailing basal inhibition in neurons}

Our recordings from cloned channels indicate that halothane caused primarily inhibition of current amplitude in homomeric channels containing HCN2 subunits, with little effect on gating; this form of inhibition could be primarily attributed to basal inhibition of channel activity imposed by the C-terminal domains of those HCN2 subunits. In channels with less basal inhibition, such as those that include HCN1 subunits (e.g., HCN1$\mathrm{HCN} 2$ or HCN1 channels), or when basal inhibition by HCN2 subunits was reduced (e.g., in the presence of cAMP), the effects of halothane were characterized more by gating shifts than by amplitude modulation. We tested whether this dependence on basal inhibition, and its modulation by cAMP, was also apparent in native neuronal $I_{\mathrm{h}}$ (Fig. 7). For this, we chose to study neurons with a relatively high ratio of $\mathrm{HCN} 2$ to $\mathrm{HCN} 1$ expression, as shown by in situ hybridization for neurons of the deep cerebellar nuclei (DCN) (Fig. 7A) (Monteggia et al., 2000; Santoro et al., 2000).

As illustrated for a DCN neuron recorded without cAMP in the pipette (Fig. $7 B$ ), the initial $V_{1 / 2}$ of $I_{\mathrm{h}}$ activation was relatively hyperpolarized (approximately $-93 \mathrm{mV}$ ), indicative of a high degree of basal inhibition, like HCN2. Accordingly, the effect of halothane on $I_{\mathrm{h}}$ in this cell was also similar to that seen with HCN2 in the absence of cAMP: halothane had little effect on $V_{1 / 2}$ but strongly decreased maximal current amplitude. In a DCN neuron recorded with cAMP in the pipette (Fig. 7C), basal inhibition was reduced (initial $V_{1 / 2}$ of approximately $-81 \mathrm{mV}$ ), and, in this case, halothane caused a strong hyperpolarizing shift in 
A

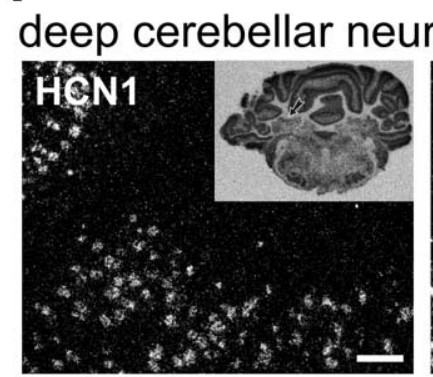

B
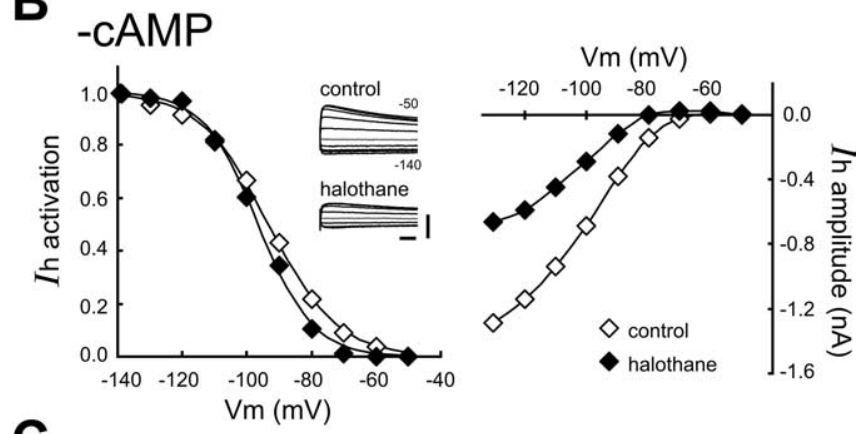

C

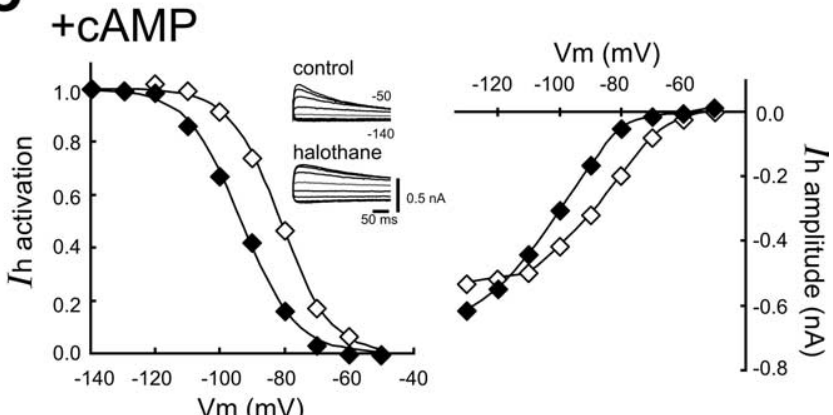

Figure 7. In DCN neurons with prominent $\mathrm{HCN} 2$ expression, the manifestation of halothane modulation depends on basal inhibition and its relief by CAMP. $\boldsymbol{A}$, In situ hybridization with $\left[{ }^{33} \mathrm{P}\right]$-labeled CRNA probes shows moderate levels of HCN1 and high levels of HCN2 transcripts in neurons of the deep cerebellar nuclei. Dark-field images of emulsion autoradiographs illustrate the localization of HCN1 and HCN2 mRNA in deep cerebellar neurons. Scale bar, $50 \mu \mathrm{m}$. Insets, Bright-field images of film autoradiographs depict representative sections. $B, C, D C N$ neurons were recorded without $\operatorname{CAMP}(B)$ and with $\operatorname{CAMP}(\boldsymbol{C})$ in the pipette; the $V_{1 / 2}$ of $I_{\mathrm{h}}$ activation (left) and steady-state $I_{\mathrm{h}}$ amplitude (right) were determined under control conditions (open diamonds) and during exposure to halothane (filled diamonds). Insets, Tail currents used for activation curves. The DCN neuron recorded without CAMP had a relatively hyperpolarized initial $V_{1 / 2}$ for $I_{h}$ activation, and halothane caused a decrease in $I_{h}$ amplitude, with little effect on $V_{1 / 2}$; the DCN neuron recorded with CAMP had a more depolarized initial $V_{1 / 2}$, and halothane caused a hyperpolarizing shift in $V_{1 / 2}$ with little amplitude inhibition.

$V_{1 / 2}$ with little effect on current amplitude, similar to its actions on HCN2 channels in the presence of cAMP.

In recordings from a population of predominantly $\mathrm{HCN} 2-$ expressing DCN and thalamocortical neurons (Monteggia et al., 2000; Santoro et al., 2000), with and without cAMP in the pipette, we obtained a distribution of initial $V_{1 / 2}$ values reflecting variable degrees of initial basal inhibition. As shown by the correlations in Figure $8 A$, the level of basal inhibition was highly predictive for the magnitude of halothane effects on gating and current amplitude. We compared neurons recorded without cAMP in the pipette that displayed high basal inhibition (initial $V_{1 / 2}$ of less than $-95 \mathrm{mV}$ ) with those recorded with cAMP that showed low basal inhibition (initial $V_{1 / 2}$ of more than $-85 \mathrm{mV}$ ). Low basal inhibition was associated with an enhanced halothane-induced shift in
A

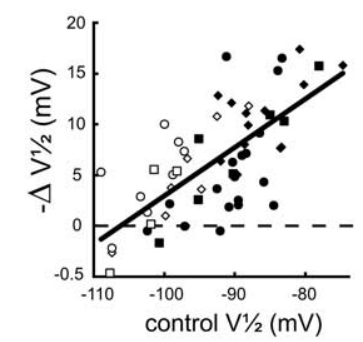

B +/- cAMP in pipette
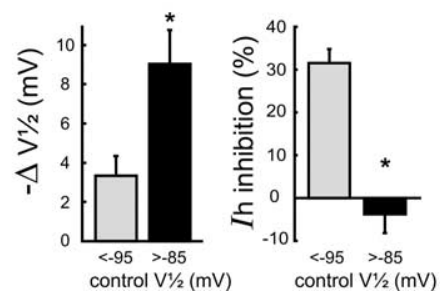

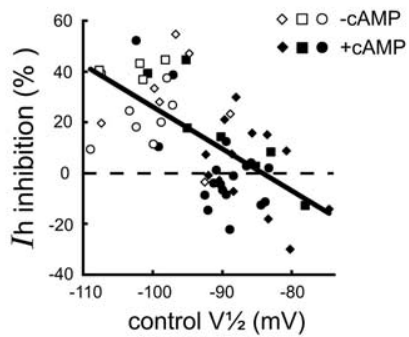

C

$\beta-A R$ stimulation

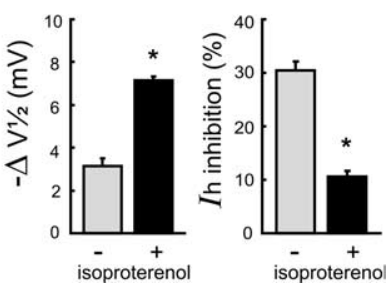

Figure 8. Elevation of intracellular CAMP in HCN2-expressing neurons alters the predominant form of $I_{\mathrm{h}}$ inhibition by halothane. $A$, The relationship between control $V_{1 / 2}$ (a measure of basal inhibition) and a halothane-induced shift in $V_{1 / 2}$ of $I_{h}$ activation (left) or effect on $I_{h}$ amplitude (right) was determined in HCN2-expressing DCN and thalamocortical neurons (DCN, diamonds; mediodorsal thalamus, squares; anterodorsal thalamus, circles). A broad range of initial $V_{1 / 2}$ values was obtained by studying cells with CAMP (0.03-1 mM; filled symbols) and without cAMP (open symbols) in the pipette. Overlaid lines indicate highly significant linear regression analysis of these data $\left(\Delta V_{1 / 2}, R^{2}=0.5, F_{(1,56)}=54.9, p<0.0001\right.$; amplitude, $R^{2}$ $\left.=0.4, F_{(1,56)}=32.2, p<0.0001\right)$. $\boldsymbol{B}$, The effect of halothane was determined in HCN2expressing $D C N$ and thalamocortical neurons recorded with or without CAMP in the pipette. In cells recorded with cAMP that had an initial $V_{1 / 2}$ of more than $-85 \mathrm{mV}(n=17)$, halothane produced a significantly greater shift in $V_{1 / 2}$ and smaller amplitude inhibition than in cells recorded without cAMP that had an initial $V_{1 / 2}$ of less than $-95 \mathrm{mV}(n=12) .{ }^{*} p<0.0001$. C, After activation of $\beta$-adrenoceptors ( $\beta$-AR) on thalamocortical neurons by isoproterenol (1 $\mu \mathrm{M}$, in the presence of $100 \mu \mathrm{m}$ IBMX), halothane caused a significantly greater shift in $V_{1 / 2}$ and smaller amplitude inhibition $(n=5) .{ }^{*} p<0.005$.

$V_{1 / 2}$ and a decreased current inhibition (Fig. $8 \mathrm{~B}$ ). Likewise, we used the $\beta$-adrenoceptor agonist isoproterenol to stimulate endogenous adenylyl cyclase activity in thalamocortical neurons with similar results; receptor-mediated increases in cAMP shifted initial $V_{1 / 2}$ (by $6.7 \pm 0.3 \mathrm{mV} ; n=5$ ) and, as shown in Figure $8 C$, coordinately increased the halothane-induced hyperpolarizing shift in $V_{1 / 2}$ while decreasing amplitude inhibition. Thus, as observed with cloned channels containing $\mathrm{HCN} 2$ subunits, elevated cAMP lowers basal inhibition of neuronal channels such that inhibition of $I_{\mathrm{h}}$ results primarily from a change in $V_{1 / 2}$ rather than a decreased current amplitude (i.e., anesthetic inhibition becomes more HCN1-like).

\section{Discussion}

Inhibition of native neuronal $I_{\mathrm{h}}$ by inhaled anesthetics is manifest in two effects, a hyperpolarizing shift in voltage dependence of activation and a decrease in maximal available current, that effectively block the current over the typical subthreshold voltage range. We show that these effects represent actions on distinct HCN channel subunits, HCN1 and HCN2, respectively, and both actions are recapitulated in $\mathrm{HCN} 1-\mathrm{HCN} 2$ heteromeric channels. Inhibition of HCN channels occurs over a clinically relevant concentration range, with near-maximal effects (approximately -10 $\mathrm{mV}$ shift in $V_{1 / 2}$ of $\mathrm{HCN} 1$ or $\sim 30 \%$ decrease in HCN2 current amplitude) at halothane concentrations $\leq 0.5 \mathrm{~mm}$. Our experiments with chimeric and deletion constructs of HCN1 and 
HCN2 indicate that inhibition by halothane of core channel activity in HCN1 and HCN2 is actually similar, but that allosteric modulation by proximal C-terminal domains dictates the form of inhibition observed. For cloned HCN channels and $I_{\mathrm{h}}$ in different neuron groups, we observed strong correlations between strength of basal inhibition, defined by initial $V_{1 / 2}$ of current activation, and either amplitude inhibition or gating shifts induced by halothane. Together, these data demonstrate that halothane modulation of native neuronal $I_{\mathrm{h}}$ is represented by more prominent gating effects in channels that include HCN1 subunits and relatively greater amplitude inhibition in channels that include HCN2 subunits; by interacting with C-terminal domains, cAMP pushes $\mathrm{HCN} 2$ channels into a state of lower basal inhibition (i.e., more depolarized $V_{1 / 2}$ ) in which actions of halothane are characterized by larger shifts in $V_{1 / 2}$ and smaller decreases in maximal available current (i.e., more HCN1-like). The strong inhibition of $\mathrm{HCN}$ channels by halothane at concentrations obtained during general anesthesia (Franks and Lieb, 1994; Eckenhoff and Johansson, 1999), to-

gether with the recognized role of $I_{\mathrm{h}}$ in CNS functions that are affected by general anesthesia (e.g., thalamocortical rhythms) (Steriade et al., 1993; McCormick and Bal, 1997; Alkire et al., 2000; Antkowiak, 2002; Harrison, 2002; Campagna et al., 2003), suggest that $I_{\mathrm{h}}$ modulation may contribute to clinical actions of these important drugs.

\section{Allosteric modulation of halothane-mediated HCN channel inhibition by C-terminal domains and cAMP}

In chimeric channels, the two forms of $I_{\mathrm{h}}$ inhibition associated with $\mathrm{HCN} 1$ or HCN2 tracked remarkably well with two distinct domains of these subunits; the proximal C-terminal region of HCN1 was necessary for halothane-induced gating shifts, whereas amplitude inhibition occurred only in chimeras that included core channel domains of HCN2. However, this seemingly strict structural demarcation was violated in the presence of cAMP, a condition that permitted halothane-induced gating shifts with little amplitude modulation in wild-type HCN2 channels or in chimeric channels that included C-terminal domains of HCN2. These results with cAMP implied that the different forms of halothane-induced channel inhibition were not attributable solely to the presence or absence of particular channel domains, a conclusion that was borne out by the demonstration that both forms of inhibition were induced simultaneously by halothane in C-terminal deletion mutants of either HCN1 or HCN2. Moreover, because inhibition by halothane of C-terminally deleted $\mathrm{HCN} 1$ and HCN2 channels was indistinguishable, distinct properties attributable to the cognate $\mathrm{C}$ termini must account for different modes of halothane-induced inhibition of intact HCN1 and HCN2 channels.

What are the known and distinct modulatory characteristics of the $\mathrm{C}$ termini of HCN1 and HCN2 channels, and how might those influence the predominant mode of inhibition by halothane? Previous mutagenesis studies demonstrated that the $\mathrm{C}$
HCN2 simulation

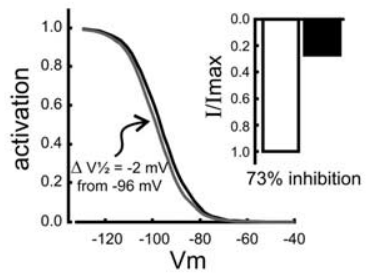

Equilibrium constants [+ halothane]

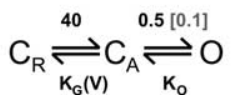

$$
\mathrm{C}_{\mathrm{R}} \underset{\mathrm{K}_{\mathrm{G}}(\mathrm{V})}{\stackrel{40}{\rightleftharpoons}} \mathrm{C}_{\mathrm{A}} \underset{\mathrm{K}_{\mathrm{o}}}{\stackrel{5[1]}{\rightleftharpoons}} \mathrm{O}
$$

HCN1-HCN2 simulation

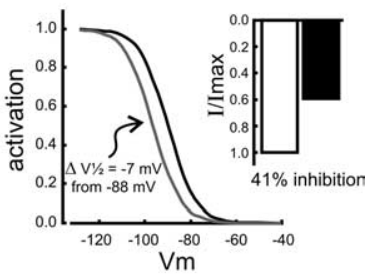

nism: stabilization of th channel closed state. In a simplified model of HCN channel gating, a fivefold decrease in the equilibrium constant $\left(K_{0}\right)$ representing a voltage-independent transition into the open state $\left(C_{A} \rightarrow 0\right)$ leads to different forms of inhibition (hyperpolarizing shift in voltage dependence or decrease in maximal current amplitude) that depends only on the initial value of that equilibrium constant. (ither a shift in voltage dependence from a relatively depolarized initial $V_{1 / 2}$, with little decrease in 作 currents were obtained with QuB freeware (http://www.qub.buffalo.edu/), and steady-state values were used to generate relative current amplitudes.

terminus of HCN2 imparts substantially more basal inhibition to its core channel domains than does the $\mathrm{C}$ terminus of $\mathrm{HCN} 1$, accounting for its more hyperpolarized initial $V_{1 / 2}$ (Wainger et al., 2001; Wang et al., 2001); binding of cAMP to the $C$ terminus acts to relieve basal inhibition, producing a more prominent depolarizing shift in $V_{1 / 2}$ of HCN2 channels. Based on a simplified model of HCN channel gating, adapted from Shin et al. (2004), we suggest that differences in C-terminally mediated basal inhibition can lead primarily to a shift in $V_{1 / 2}$ or amplitude inhibition despite a common underlying inhibitory mechanism, i.e., stabilization of channel closed state.

This concept is illustrated in Figure 9, in which currents were simulated using a three-state model: a resting closed state $\left(C_{R}\right)$ is separated from an activated closed state $\left(\mathrm{C}_{\mathrm{A}}\right)$ by a voltagedependent gating transition with equilibrium constant $K_{\mathrm{G}}(V)$, and a second transition from $\mathrm{C}_{\mathrm{A}}$ to the conducting open state $(\mathrm{O})$ is defined by a voltage-independent equilibrium constant $\left(K_{\mathrm{O}}\right)$. For this simulation, we used an identical $K_{\mathrm{G}}(V)$ for both HCN1 and $\mathrm{HCN} 2$, and we assigned 100-fold differences in the magnitude of $K_{\mathrm{O}}$ to account for differences in C-terminally mediated basal inhibition in the subunits. With these parameters, the initial $V_{1 / 2}$ is more hyperpolarized in the model with 100 -fold smaller $K_{\mathrm{O}}$ (by approximately $-21 \mathrm{mV}$ ), similar to differences observed between HCN1 and HCN2 channels. Under these different initial conditions, simulating channel modulation by halothane by applying identical fivefold decreases in $K_{\mathrm{O}}$ to both models leads to apparently different forms of inhibition: with the higher initial $K_{\mathrm{O}}$, inhibition predominantly appears as a hyperpolarizing shift in $V_{1 / 2}$ (like $H C N 1$ ), whereas with the lower initial $K_{\mathrm{O}}$, inhibition is primarily manifest as a decrease in maximal current amplitude (like HCN2). With an intermediate initial $K_{\mathrm{O}}$, the initial $V_{1 / 2}$ is also intermediate and the same fivefold decrease yields both forms of inhibition (like HCN1-HCN2). To account for the ability of cAMP to change halothane-induced modulation of HCN2 
and $\mathrm{HCN} 1-\mathrm{HCN} 2$ channels to a more HCN1-like pattern (i.e., gating shift with little amplitude inhibition), one need only propose that binding of cAMP decreases basal inhibition (increases initial $K_{\mathrm{O}}$ ) to approximate that of $\mathrm{HCN1}$, as has been demonstrated (Wainger et al., 2001; Wang et al., 2001).

These arguments for distinct manifestations of channel inhibition are very similar to those proposed to account for different forms of activation induced by cAMP in two HCN channels (Shin et al., 2004), although in that previous model, an inactivated state was introduced to account for the somewhat anomalous behavior of a sea urchin $\mathrm{HCN}$ channel. In addition, an analogous situation obtains in the related cyclic nucleotide-gated (CNG) channels (Fodor et al., 1997). Inhibition of olfactory CNG channels by the local anesthetic tetracaine involved a shift in concentration dependence for cGMP, with little effect on current amplitude, whereas tetracaine strongly decreased maximal current amplitude in rod CNG channels; the relative expression of these two forms of inhibition was ascribed to differences in $K_{\mathrm{O}}$ intrinsic to the two CNG channels (Fodor et al., 1997).

We should note, however, some deviations in the data that suggest complexities in gating of chimeric channels that are not easily explained by our simple model, at least by its use of initial $V_{1 / 2}$ as a measure of basal inhibition (and $K_{\mathrm{O}}$ ). For example, halothane had minimal effects on $V_{1 / 2}$ or current amplitude in chimeras combining core domains from $\mathrm{HCN} 1$ with proximal $\mathrm{C}$ termini from HCN2, whereas it produced strong inhibition of maximal current and a pronounced gating shift in constructs containing HCN2 core domains and HCN1 C-terminal regions, despite similar initial $V_{1 / 2}$ values (see supplemental data, available at www.jneurosci.org as supplemental material). Also, for those chimeras in which both inhibitory effects were evident (e.g., those that combine an $\mathrm{HCN} 2$ core with proximal C-terminal regions of $\mathrm{HCN} 1$ ), halothane continued to cause a strong decrease in current amplitude even when the initial $V_{1 / 2}$ was shifted to more depolarized potentials in the presence of cAMP (see Fig. $5 G$ ). Of course, domain functions are unlikely to be completely interchangeable in chimeric channels, and these discrepancies may reflect altered conformational coupling from CNBD to $\mathrm{C}$ linker to the channel pore (Craven and Zagotta, 2004). In this respect, the cAMP-bound $\mathrm{HCN} 1 \mathrm{C}$ terminus does not appear to modulate the HCN2 core to the same extent as does the natural cAMP-bound HCN2 C terminus (see also Wang et al., 2001). In addition, our model does not attempt to account for any differences in the voltage-dependent transition $\left[K_{\mathrm{G}}(V)\right]$ that could result from domain mismatches in chimeric channels and, for example, in electrostatic interactions between C-linker and S4-S5 linker domains that affect voltage dependence of $\mathrm{HCN}$ channels (Decher et al., 2004). Nevertheless, the overall correlation between initial $V_{1 / 2}$ and either halothane-induced gating shifts or amplitude inhibition was upheld across the group of mutant HCN channels and, more importantly, was recapitulated in neurons expressing native $I_{\mathrm{h}}$ channels. Moreover, the simple model illustrates a key point: a single mechanism can result in apparently distinct forms of inhibition of HCN channels.

\section{Potential functional consequences of $\mathrm{HCN}$ channel modulation by anesthetics}

We have shown that halothane inhibition of $I_{\mathrm{h}}$ in neurons can include a shift in activation voltage, inhibition of current amplitude, or both, depending on HCN subunit composition and degree of tonic cAMP modulation. HCN channels represent a particularly provocative potential anesthetic target because subtle modulation of channel activity, as observed with physiological changes in cAMP levels, can contribute to substantially altered rhythmic activity patterns that accompany various behavioral states (e.g., the slow EEG rhythms of sleep, when $I_{\mathrm{h}}$ is inhibited in thalamocortical neurons or the tachycardia of exercise, when sinoatrial nodal $I_{\mathrm{f}}$ is enhanced) (DiFrancesco, 1993; Pape, 1996; Clapham, 1998; Lüthi and McCormick, 1998). Moreover, although $I_{\mathrm{h}}$ is best known for its contributions to cellular pacemaker behavior, the current is also present in many nonpacemaker neurons in which it can affect integrative properties in numerous ways: $I_{\mathrm{h}}$ can stabilize resting membrane potential, mediate rebound depolarization and hyperpolarization, contribute to spike repolarization, decrease the amplitude and time course of spike afterhyperpolarization, and modify dendritic filtering of synaptic inputs (for review, see Pape, 1996). It is likely, therefore, that modulation of $I_{\mathrm{h}}$ by anesthetics in pacemaker and nonpacemaker cells can profoundly affect neuronal excitability, and we suggest that this modulation can contribute to clinical actions of volatile anesthetic agents. It will be important to determine, by using knock-out animals and/or computational models, how differential inhibition by anesthetics of either voltage dependence of activation or current amplitude, mediated by HCN1 or HCN2 subunits and modulated by cAMP, ultimately influences the actions of those anesthetics.

\section{References}

Alkire MT, Haier RJ, Fallon JH (2000) Toward a unified theory of narcosis: brain imaging evidence for a thalamocortical switch as the neurophysiologic basis of anesthetic-induced unconsciousness. Conscious Cogn 9:370-386.

Antkowiak B (2002) In vitro networks: cortical mechanisms of anaesthetic action. Br J Anaesth 89:102-111.

Biel M, Ludwig A, Zong X, Hofmann F (1999) Hyperpolarization-activated cation channels: a multi-gene family. Rev Physiol Biochem Pharmacol 136:165-181.

Campagna JA, Miller KW, Forman SA (2003) Mechanisms of actions of inhaled anesthetics. N Engl J Med 348:2110-2124.

Chen S, Wang J, Siegelbaum SA (2001) Properties of hyperpolarizationactivated pacemaker current defined by coassembly of HCN1 and HCN2 subunits and basal modulation by cyclic nucleotide. J Gen Physiol 117:491-504.

Clapham DE (1998) Not so funny anymore: pacing channels are cloned. Neuron 21:5-7.

Craven KB, Zagotta WN (2004) Salt bridges and gating in the COOHterminal region of $\mathrm{HCN} 2$ and CNGA1 channels. J Gen Physiol 124:663-677.

Decher N, Chen J, Sanguinetti MC (2004) Voltage-dependent gating of hyperpolarization-activated cyclic nucleotide-gated pacemaker channels: molecular coupling between the S4-S5 and C-linkers. J Biol Chem 279:13859-13865.

DiFrancesco D (1993) Pacemaker mechanisms in cardiac tissue. Annu Rev Physiol 55:455-472.

Eckenhoff RG, Johansson JS (1999) On the relevance of "clinically relevant concentrations" of inhaled anesthetics in in vitro experiments. Anesthesiology 91:856-860.

Fodor AA, Gordon SE, Zagotta WN (1997) Mechanism of tetracaine block of cyclic nucleotide-gated channels. J Gen Physiol 109:3-14.

Franks NP, Lieb WR (1988) Volatile general anaesthetics activate a novel neuronal $\mathrm{K}^{+}$current. Nature 333:662-664.

Franks NP, Lieb WR (1994) Molecular and cellular mechanisms of general anaesthesia. Nature 367:607-614.

Harrison NL (2002) General anesthesia research: aroused from a deep sleep? Nat Neurosci 5:928-929.

Harrison NL (2003) Knockin' on the door of general anesthetic mechanisms: but will U.S. researchers be shut out? Anesth Analg 97:616-618.

Heurteaux C, Guy N, Laigle C, Blondeau N, Duprat F, Mazzuca M, LangLazdunski L, Widmann C, Zanzouri M, Romey G, Lazdunski M (2004) TREK-1, a K ${ }^{+}$channel involved in neuroprotection and general anesthesia. EMBO J 23:2684-2695.

Jurd R, Arras M, Lambert S, Drexler B, Siegwart R, Crestani F, Zaugg M, Vogt 
KE, Ledermann B, Antkowiak B, Rudolph U (2003) General anesthetic actions in vivo strongly attenuated by a point mutation in the $\mathrm{GABA}_{\mathrm{A}}$ receptor $\beta 3$ subunit. FASEB J 17:250-252.

Kaupp UB, Seifert R (2001) Molecular diversity of pacemaker ion channels. Annu Rev Physiol 63:235-257.

Krasowski MD, Harrison NL (1999) General anaesthetic actions on ligandgated ion channels. Cell Mol Life Sci 55:1278-1303.

Lüthi A, McCormick DA (1998) H-current: properties of a neuronal and network pacemaker. Neuron 21:9-12.

McCormick DA, Bal T (1997) Sleep and arousal: thalamocortical mechanisms. Annu Rev Neurosci 20:185-215.

Monteggia LM, Eisch AJ, Tang MD, Kaczmarek LK, Nestler EJ (2000) Cloning and localization of the hyperpolarization-activated cyclic nucleotidegated channel family in rat brain. Brain Res Mol Brain Res 81:129-139.

Moosmang S, Biel M, Hofmann F, Ludwig A (1999) Differential distribution of four hyperpolarization-activated cation channels in mouse brain. Biol Chem 380:975-980.

Much B, Wahl-Schott C, Zong X, Schneider A, Baumann L, Moosmang S, Ludwig A, Biel M (2003) Role of subunit heteromerization and N-linked glycosylation in the formation of functional hyperpolarization-activated cyclic nucleotide-gated channels. J Biol Chem 278:43781-43786.

Pape HC (1996) Queer current and pacemaker: the hyperpolarizationactivated cation current in neurons. Annu Rev Physiol 58:299-327.

Patel AJ, Honore E, Lesage F, Fink M, Romey G, Lazdunski M (1999) Inhalational anesthetics activate two-pore-domain background $\mathrm{K}^{+}$channels. Nat Neurosci 2:422-426.

Rekling JC, Funk GD, Bayliss DA, Dong XW, Feldman JL (2000) Synaptic control of motoneuronal excitability. Physiol Rev 80:767-852.

Reynolds DS, Rosahl TW, Cirone J, O’Meara GF, Haythornthwaite A, Newman RJ, Myers J, Sur C, Howell O, Rutter AR, Atack J, Macaulay AJ, Hadingham KL, Hutson PH, Belelli D, Lambert JJ, Dawson GR, McKernan R, Whiting PJ, Wafford KA (2003) Sedation and anesthesia mediated by distinct $\mathrm{GABA}_{\mathrm{A}}$ receptor isoforms. J Neurosci 23:8608-8617.

Rudolph U, Mohler H (2004) Analysis of $\mathrm{GABA}_{\mathrm{A}}$ receptor function and dissection of the pharmacology of benzodiazepines and general anesthetics through mouse genetics. Annu Rev Pharmacol Toxicol 44:475-498.
Santoro B, Tibbs GR (1999) The HCN gene family: molecular basis of the hyperpolarization-activated pacemaker channels. Ann NY Acad Sci 868:741-764.

Santoro B, Chen S, Luthi A, Pavlidis P, Shumyatsky GP, Tibbs GR, Siegelbaum SA (2000) Molecular and functional heterogeneity of hyperpolarizationactivated pacemaker channels in the mouse CNS. J Neurosci 20:5264-5275.

Shin KS, Maertens C, Proenza C, Rothberg BS, Yellen G (2004) Inactivation in $\mathrm{HCN}$ channels results from reclosure of the activation gate: desensitization to voltage. Neuron 41:737-744.

Sirois JE, Pancrazio JJ, Lynch III C, Bayliss DA (1998) Multiple ionic mechanisms mediate inhibition of rat motoneurones by inhalation anaesthetics. J Physiol (Lond) 512:851-862.

Sirois JE, Lynch III C, Bayliss DA (2002) Convergent and reciprocal modulation of a leak $\mathrm{K}^{+}$current and $I_{\mathrm{h}}$ by an inhalational anaesthetic and neurotransmitters in rat brainstem motoneurones. J Physiol (Lond) 541:717-729.

Sonner JM, Antognini JF, Dutton RC, Flood P, Gray AT, Harris RA, Homanics GE, Kendig J, Orser B, Raines DE, Rampil IJ, Trudell J, Vissel B, Eger EI (2003) Inhaled anesthetics and immobility: mechanisms, mysteries, and minimum alveolar anesthetic concentration. Anesth Analg 97:718-740.

Steriade M, McCormick DA, Sejnowski TJ (1993) Thalamocortical oscillations in the sleeping and aroused brain. Science 262:679-685.

Talley EM, Bayliss DA (2002) Modulation of TASK-1 (Kcnk3) and TASK-3 (Kcnk9) potassium channels: volatile anesthetics and neurotransmitters share a molecular site of action. J Biol Chem 277:17733-17742.

Ulens C, Tytgat J (2001) Functional heteromerization of HCN1 and HCN2 pacemaker channels. J Biol Chem 276:6069-6072.

Wainger BJ, DeGennaro M, Santoro B, Siegelbaum SA, Tibbs GR (2001) Molecular mechanism of cAMP modulation of $\mathrm{HCN}$ pacemaker channels. Nature 411:805-810.

Wang J, Chen S, Siegelbaum SA (2001) Regulation of hyperpolarizationactivated HCN channel gating and cAMP modulation due to interactions of $\mathrm{COOH}$ terminus and core transmembrane regions. J Gen Physiol 118:237-250.

Zagotta WN, Olivier NB, Black KD, Young EC, Olson R, Gouaux E (2003) Structural basis for modulation and agonist specificity of HCN pacemaker channels. Nature 425:200-205. 